\title{
PERFORMANCE OF CHEST COMPRESSIONS WITH THE USE OF THE NEW MECHANICAL CHEST COMPRESSION MACHINE LIFELINE ARM: A RANDOMIZED CROSSOVER MANIKIN STUDY IN NOVICE PHYSICIANS
}

\author{
Sebastian Aleksandrowicz ${ }^{1}$, Marcin Madziala², Lukasz Iskrzycki ${ }^{3}$, \\ Zenon Truszewski ${ }^{2}$ Pawel Gawlowski ${ }^{3}$ \\ ${ }^{1}$ Polish Society of Disaster Medicine, Poland \\ ${ }^{2}$ Department of Emergency Medicine, Medical University of Warsaw, Warsaw, Poland \\ ${ }^{3}$ Department and Emergency Medical Service, Wroclaw Medical University, Wroclaw, Poland
}

\begin{abstract}
BACKGROUND: The Lifeline ARM (ARM; Defibtech, Guilford, USA) is a new mechanical chest compression device. The aim of the current study was to compare the quality of single rescuer cardiopulmonary resuscitation (CPR) with and without ARM device.
\end{abstract}

METHODS: In this randomized crossover manikin trial forty-four novice physicians participated. Thirty minutes of training was allotted for manual CPR and then for the ARM. The following day, every participant performed a 2-min CPR single rescuer scenario, once with manual CPR and once with the ARM. The primary outcome measure of the study is effective compression; defined as compressions performed with the correct of depth of 50-60 mm, complete decompressions, and the correct pressure point of CC.

RESULTS: The ARM, compared with manual CPR, carried out more effective compressions (96 [interquartile range, IQR; 94-98] vs. 36 [IQR; 33-41]\%, $\mathrm{p}<0.001)$. The compressions preformed with the use of the ARM, furthermore, were with a correct CC rate (100 [IQR; 99-101] vs. 130 [IQR; 124-140] $\left.\mathrm{min}^{-1} ; \mathrm{p}<0.001\right)$ and a correct depth (97 [IQR; 96-98] vs. 37 [IQR; 31-39]\%; $p<0.001$ ). The result of resuscitation with ARM was significantly better than manual CPR $(p<0.05)$ for all of the analyzed chest compression parameters (percentage of CC too deep, percentage of $\mathrm{CC}$ too shallow, percentage of correct pressure points and percentage of correct pressure releases), as well as for the ventilation parameters (tidal volume, ventilation rate, minute-volume, gastric inflations).

CONCLUSION: During this simulated trial, when CPR was performed by novice physicians, the ARM significantly improved the quality of CPR. Further clinical trials should provide motivation to confirm the potential benefits of ARM use during CPR.

KEY WORDS: cardiopulmonary resuscitation, chest compression, quality, physician, simulation

Disaster Emerg Med J 2016; (1)1: 30-36

\section{INTRODUCTION}

Sudden cardiac arrest (SCA) is the leading cause of death in Europe, with an overall resuscitation success rate less than $10 \%$ despite widespread train- ing in cardiopulmonary resuscitation (CPR) and defibrillation [1, 2]. Although survival from cardiac arrest requires high-quality CPR $[3,4]$, some studies demonstrate that the quality of CPR performance 
during SCA is poor, even when CPR is conducted by medical staff [5-9]. Moreover, CPR quality becomes ineffective within minutes due to rescuer fatigue $[10,11]$.

The current European Resuscitation Council (ERC) 2015 guidelines, demonstrate that automated mechanical chest compression devices are a reasonable alternative to high-quality manual chest compressions in situations where sustained high-quality manual chest compressions are impractical or compromise rescuer safety $[12,13]$. In studies by Xanthos et al., the use of mechanical chest compression devices minimized resuscitation-related trauma compared with manual chest compressions in a swine model of cardiac arrest [14]. Several studies, furthermore, show promising results in regard to the survival and return of spontaneous circulation (ROSC) with the use of mechanical chest compression devices [15].

The Lifeline ARM (Defibtech, Guilford, CT, USA) mechanical chest compression device is an automated, portable, battery or AC-powered device that provides mechanical chest compressions on adult patients in cardiac arrest. The device is designed either for high quality CPR performed by medical personnel and laypersons in out-of-hospital or in in-hospital CPR settings. The current study compared the quality of single rescuer resuscitation CPR with and without the use of ARM during resuscitation with the hypothesis that the use of the ARM during CPR would improve the performance of chest compressions and ventilations performed by novice physicians.

\section{MATERIAL AND METHODS}

\section{Study design and setting}

This open, prospective, randomized, crossover manikin study was approved by the Institute Review Board of the International Institute of Rescue Research and Education (approved: 03.2016.04.11 on March $\left.25^{\text {th }}, 2016\right)$. The study was conducted between May and June 2016 in accordance with the Declaration of Helsinki.

\section{Participants}

Based on pilot data, we assumed an alpha risk of 0.05 , and a beta risk of 0.2 for the sample size calculation. The percentage of effective compressions in the pilot data with the use of manual CPR and
ARM varied and amounted to $44.3 \%$ vs. $100 \%$, respectively (standard BLS [basic life support] and ARM). We calculated that 31 participants would be required (paired, two-sided). The participants were randomized with a 1:1 ratio.

With voluntary written informed consent, 44 novice physicians were recruited who satisfied the following inclusion criteria: (1) they had not used the mechanical chest compression devices before the study; and (2) they presented no wrist or lower back conditions, or pregnancy. The study was conducted between May and June 2016 in accordance with the Declaration of Helsinki.

\section{Device}

The Lifeline ARM (Defibtech, Guilford, CT, USA) Automated Chest Compression (ACC) system is a mechanical solution for providing quality CPR (recommended rate and depth with complete chest wall recoil between compressions) using a proprietary software algorithm that compensates for variability in patient chest resistances, which can change during the rescue. The portable device is battery- or AC-powered, and may be used under a variety of circumstances, both in and out of the hospital, on adult victims of sudden cardiac arrest (SCA; Fig. 1). The device accommodates a wide range of adult patient size extremes. Deployed by qualified medical personnel and trained rescuers certified to administer CPR, such as professional first responders and healthcare providers, it provides effective CPR without interruptions. The Lifeline ARM delivers chest

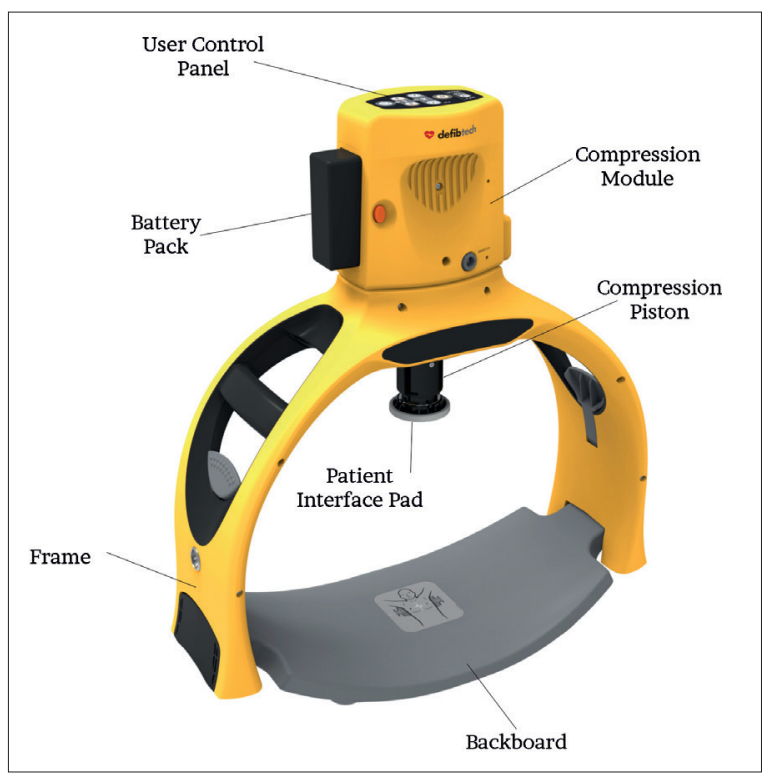

FIGURE 1. The Lifeline ARM chest compression device 


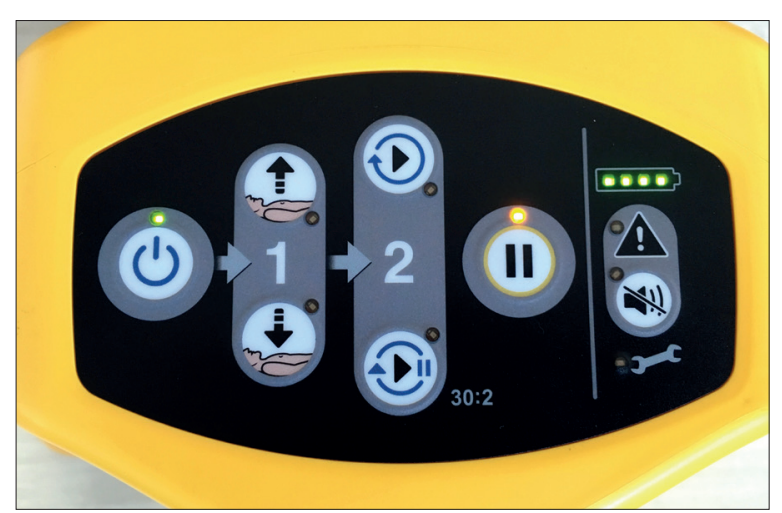

FIGURE 2. The Lifeline ARM user interface

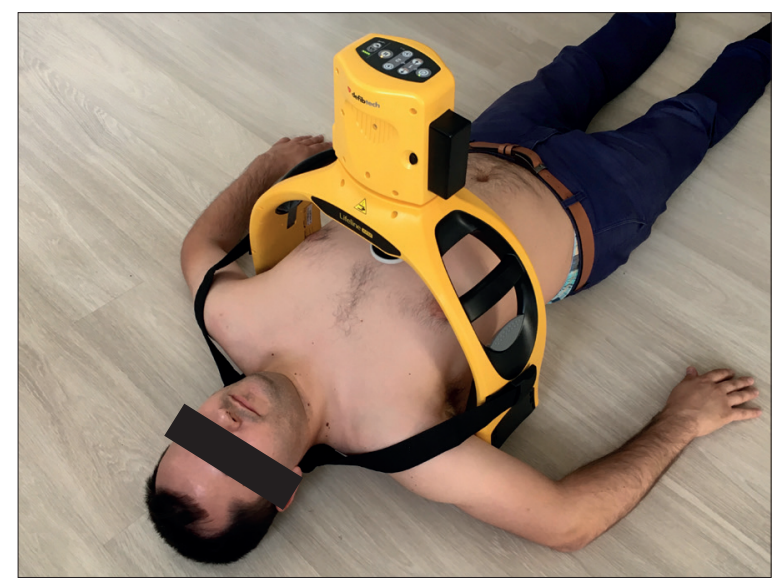

FIGURE 3. The Lifeline ARM device on a patient

compressions using a software controlled compression piston powered by a direct drive motor housed in a removable compression module. Modularization of the compression piston enables deployment, product maintenance, and serviceability. The module also contains the user interface (Fig. 2) and battery technology. Power options allow for prolonged CPR efforts which may be beneficial. Patient accessibility throughout deployment and operation are provided by a frame with self-centring and self-locking latching mechanisms and two sets of wide release levers that are incorporated into each side of the frame along with patient lift handles (Fig. 3). During operation, the structural integrity of the single-piece design of the frame paired with a backboard allow chest compressions without undue deflection or distortion, factors which are important regarding CPR efficacy.

\section{Study protocol}

Prior to the study, all participants attended in a 2-hour lecture concerning the theoretical knowl- edge of cardiopulmonary resuscitation, which included, basic and advanced life support guidelines in accordance to the European Resuscitation Council guidelines of 2015 [1]. After the lecture, a demonstration with instruction of cardiopulmonary resuscitation, with and without the use of the ARM device, was presented to all participants. All participants then practiced BLS methods for 30 minutes. Subsequently, in order to ensure participants were familiar with the proper use of the ARM, all participants used the ARM device.

The next day, using Research Randomizer software (randomizer.org) participants were divided into two groups: the first group started resuscitation without the ARM, and the second with the ARM. Each group performed 2-min cardiopulmonary resuscitation in a single rescuer condition, on the floor under direct supervision of the investigator.

During CPR without ARM, the rescuer was positioned at the side of the manikins' chest and changed position for the delivery of rescue breaths. However, during CPR with ARM, after turning the ARM on the rescuer was positioned at the side of the manikins' head the entire time. After finishing the given group's resuscitation scenario, participants had a 20 minute break and then performed CPR employing the other method. The detailed randomization of participants is presented in Figure 4.

\section{Outcome measures}

The primary outcome of the study is the number of effective compressions, which is defined as compressions performed with the correct depth of 50$-60 \mathrm{~mm}$, complete decompression, and the correct pressure point of CC.

Secondary outcomes comprise the mean depth and rate of chest compressions, an incorrect pressure point and incomplete decompressions, as well as, ventilator parameters such as tidal volume, ventilation rate, minute-volume, and number of gastric inflations. Also recorded is the "absolute hands-off time", defined as the sum of all periods during which no hand was placed on the chest minus time used for ventilation (ventilation time). The trial manikin was connected to a computer that allowed the chest compression and ventilation parameters to be collected using Skill Reporting software version 2.0.0.14 (Resusci Anne Skill Reporter, Laerdal Medical, Stavanger, Norway). While performing CPR, the participants were not provided with any information recorded by the manikin monitoring system and 


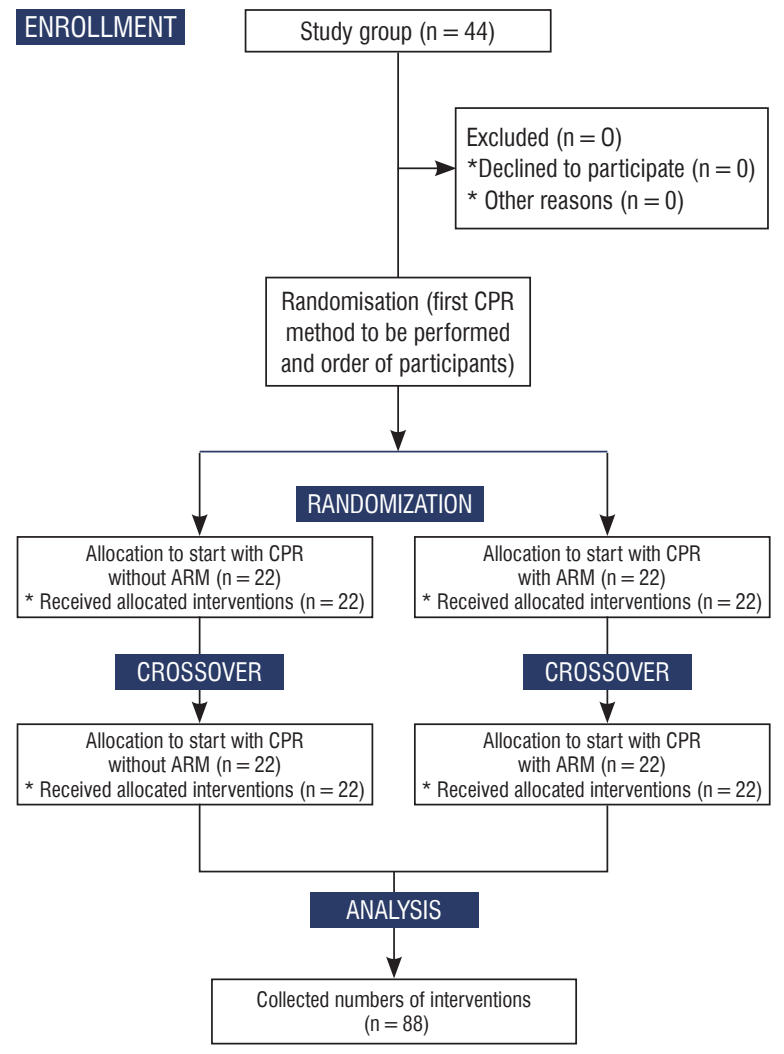

CPR — cardiopulonary resuscitation, ARM — LifeLine ARM device

FIGURE 4. CONSORT flow-charts

were guided only by their own experience. After the end of the CPR procedure, each participant was asked to complete a questionnaire concerning the ease of use and their personal level of confidence while using the ARM. The age, gender, body weight, height, and body mass index (BMI) of all participants was also collected.

\section{Statistical analysis}

The results were presented as absolute values, percentages, median and interquartile range (IQR), or mean and standard deviation (SD). The Statistica software package (version 12.5, StatSoft Inc., Tulsa, OK, USA) was used for statistical analysis. The Kolmogorov-Smirnov test was applied to check for normal distribution. As this was a randomized crossover trial, pairing was taken into account in the statistical analysis. The Student t test was used for paired samples with a normal distribution, and the Wilcoxon test for samples with a non-parametric distribution. All $p$ values were two-sided and $p<0.05$ was considered to be statistically significant. In the comparative analysis of chest compression depth, as well as the personal variables [weight, height, Body Mass
Index (BMI), sex], simple linear regression analysis Pearson's correlation was applied in order to detect and describe the strength and direction of correlations of CC depth to the above body composition data.

\section{RESULTS}

44 novice physicians (16 female; $36.4 \%$ ) participated in the trial. Their mean age was $27.4 \pm 1.9$ years, mean height was $170 \pm 9 \mathrm{~cm}$, while mean weight was $76.5 \pm 10.5 \mathrm{~kg}$. The full characteristics of the participants are presented in Table 1.

Effective compressions during resuscitation with the ARM and in manual CPR varied and amounted to: 96 (interquartile range, IQR; 94-98) vs. 36 (33-41)\% (Tab. 2). The median correct depth of CC was significantly different between trials with the ARM and manual CPR (97 vs. 37\%, respectively; $p<0.001$ ). The median CC rate varied significantly between trials with the ARM and manual CPR $\left(100 \mathrm{~min}^{-1}\right.$ with ARM vs. $130^{-1}$ without $\left.A R M ; p<0.001\right)$. The results demonstrate that resuscitation performed with the ARM was significantly better than in manual CPR $(p<0.05)$ for all the analyzed chest compression parameters (percentage of CC too deep, percentage of CC too shallow, percentage of correct pressure points and percentage of correct pressure releases).

During resuscitation with the ARM, notably more rescue breaths reached the correct ventilation volume as compared with resuscitation without ARM (Tab. 2). In addition, with the use of the ARM percentage of gastric inflations was statistically less than in resuscitation without the ARM $(p<0.001)$.

A simple regression analysis showed that the male gender was a significant factor in the depth of the chest compression $(r=0.32 ; p=0.017)$, as well as, weight $(r=0.34 ; p=0.012)$. Other variables (weight, BMI) were not significantly associated with CC depth during resuscitation without ARM $(r=0.13, p=0.052$ for weight; $r=0.18$,

\begin{tabular}{|l|c|}
\hline \multicolumn{2}{|l|}{ Table 1. Participant characteristics } \\
\hline $\begin{array}{l}\text { Gender } \\
\text { Male } \\
\text { Female }\end{array}$ & $28(63.6 \%)$ \\
$16(36.4 \%)$ \\
\hline Age & $27.4 \pm 1.9$ \\
\hline Height $[\mathrm{cm}]$ & $170 \pm 9$ \\
\hline Weight $[\mathrm{kg}]$ & $76.5 \pm 10.5$ \\
\hline BMl & $22.5 \pm 1.7$ \\
\hline
\end{tabular}




\begin{tabular}{|c|c|c|c|}
\hline & Manual CPR & ARM & $\mathrm{p}$-value \\
\hline \multicolumn{4}{|c|}{ Chest compression parameters } \\
\hline Effective compressions [\%] & $36(33-41)$ & $96(94-98)$ & $<0.001$ \\
\hline Correct CC depth [\%] & $37(31-39)$ & $97(96-98)$ & $<0.001$ \\
\hline CC too deep [\%] & $24(21-26)$ & $2(1-3)$ & $<0.001$ \\
\hline CC too shallow [\%] & $41(35-53)$ & $1(1-2)$ & $<0.001$ \\
\hline Median CC rate $\left(\mathrm{min}^{-1}\right)$ & $130(124-140)$ & $100(99-101)$ & $<0.001$ \\
\hline Median CC depth [mm] & $35(30-37)$ & $52(51-53)$ & $<0.001$ \\
\hline Correct pressure point [\%] & 85 (67-94) & $100(99-100)$ & 0.003 \\
\hline Correct pressure release [\%] & $73(63-90)$ & $100(99-100)$ & $<0.001$ \\
\hline \multicolumn{4}{|c|}{ Ventilation parameters } \\
\hline Tidal volume [l] & $0.34(0.28 \pm 0.37)$ & $0.5(0.43-0.51)$ & $<0.001$ \\
\hline Ventilation rate [min] & $3(2-4)$ & $6(5-7)$ & $<0.001$ \\
\hline Minute-volume [I] & $1.04(0.91-1.5)$ & $2.9(2.3-3.5)$ & $<0.001$ \\
\hline Gastric inflations [\%] & $6(4-10)$ & $1(1-2)$ & $<0.001$ \\
\hline \multicolumn{4}{|c|}{ Time-related parameters } \\
\hline Absolute hands-off time [s] & $32(29-38)$ & $18(17-19)$ & $<0.001$ \\
\hline
\end{tabular}

$\mathrm{p}=0.23$ for $\mathrm{BMI}$ ). None of the variables (gender, weight, height, BMI) was significantly associated with CC depth during CPR with the use of the ARM.

All participants, in their subjective opinion, judged that ARM helped them in performing CPR with $100 \%$ of participants stating that they would use the ARM in a real cardiac arrest situation. Furthermore, $95.5 \%$ of participants felt more confident in performing CPR using the ARM.

\section{DISCUSSION}

In this trial on simulated CPR performed by novice physicians, the ARM device significantly increased the CC quality, improving the CC depth and rate, and the correct degree of chest relaxation in comparison with manual CPR. Moreover, the use of ARM allowed one to obtain better ventilation parameters during CPR.

Effective CCs, with the appropriate depth and minimization of interruptions, are the gold standard according to the ERC 2015 guidelines for CPR. Currently, there are no clear recommendations with regard to the routine use of mechanical CC systems. However, according to the ILCOR guidelines, these devices are applied if high-quality CPR is unavailable, including cases of CPR provided by untrained medical personnel. Mechanical CC devices have been developed to better deliver good quality uninterrupted CCs [16] and may also be preferred during transportation $[17,18]$, in a catheterization laboratory, and as a bridge to more invasive support such as extracorporeal membrane oxygenation $[19,20]$.

The devices ensure effective CPR with the CC depth (at least 2 inches $/ 5 \mathrm{~cm}$ ) and rate (at least 100 per minute) as recommended in the current AHAJERC guidelines $[1,21]$. The analysis of the study material proves that the use of the ARM during a 2-min CPR cycle resulted in a statistically significantly greater CC depth, as well as a CC frequency consistent with the current guidelines. It is worth noticing that recent studies found a strong association between survival outcomes and increased CC depth [22].

In several studies, the CCs performed by medical personnel turned out too shallow or too deep $[10,23]$. In a research by Peberdy et al., the participants provided too shallow CCs in $34 \%$ of the attempts, whereas the CCs in $12 \%$ of the attempts were too deep [24].

With manual CPR, the median CC rate was 130 (IQR, 124-140) $\mathrm{min}^{-1}$. Sunde et al. suggested that too high rates might lead to a decrease in the CC depth and impair diastolic filling and coronary perfusion owing to insufficient decompression [25]. The ARM device is programmed to perform CCs with an adequate rate and chest recoil. Several authors 
indicate that chest recoil during manual CPR is insufficient $[10,18,23,26]$. The ARM devices provide high-quality CCs throughout the scenario.

During CPR, adequate patient ventilation constitutes another key point. The oxygen reserve during sudden cardiac arrest in normothermia patients is sufficient for merely 3-5 minutes; after this time, hypoxia leads to irreversible changes to the central nervous system $[1,6]$. When novice physicians used ARM, ventilation parameters were better than in the manual CPR scenario.

In the prehospital settings, each patient should be treated as a patient with a full stomach. Gastric inflation is an important complication of rescue breaths, and can lead to aspiration and ARDS. The reduced risk of gastric inflation when using ARM in clinical practice may result in higher patient safety with better pulmonary gas exchange.

Both the CC quality and shorter hands-off time directly influence survival during CPR $[27,28]$. In the present study, the 'absolute hands-off time', defined as the sum of all periods during which no hand is placed on the chest minus the time used for ventilation with ARM, was 18 (IQR, 17-19) seconds, and turned out to be significantly shorter than in manual CPR conditions (32 [IQR, 29-38] seconds; $p<0.001)$. This relationship is confirmed by, among others, Putzer et al. [29, 30] and Fischer et al. [26]. In these studies, the use of mechanical CC devices also reduced the hands-off time as compared with manual CPR.

\section{LIMITATIONS}

Firstly, the trial was conducted on a manikin, not in clinical conditions. Although the setting does not necessarily reflect real life CPR situations, on the other hand, manikins are standardized, while patients represent a great variety of conditions. Thus, conducting the study on a manikin allowed one to standardize the CPR setting. Moreover, according to the ILCOR decision, randomized crossover trials during resuscitation are unethical. The second limitation is the fact that only novice physicians participated in the study. However, owing to their limited experience in conducting CPR, this group may require applying devices to improve the CPR effectiveness. This was a randomized, crossover manikin trial; although it was not possible to blind the participants to the intent of the study, they were blinded to the adequacy of CC parameters during the CPR procedure.
Further investigations, especially clinical studies, are needed to evaluate the obtained results.

\section{CONCLUSIONS}

In this simulated trial, when CPR was performed by novice physicians, the ARM significantly improved the quality of CPR. Further clinical trials should provide motivation to confirm the potential benefits of ARM use during CPR.

\section{Compliance with Ethical Standards \\ Funding: None.}

Conflicts of interest: The authors have no conflicts of interest to disclose.

Ethical approval: This open, prospective, randomized, crossover trial was approved by the Institutional Review Board of International Institute of Rescue Research and Education.

Informed consent: Informed consent was obtained from all individual participants included in the study.

\section{Acknowledgments}

We would like to thank all physicians for their participation in our study.

\section{REFERENCES}

1. Perkins GD, Handley AJ, Koster RW et al. Adult basic life support and automated external defibrillation section Collaborators. European Resuscitation Council Guidelines for Resuscitation 2015: Section 2. Adult basic life support and automated external defibrillation. Resuscitation, 2015; 95: 81-99. doi: 10.1016/j.resuscitation.2015.07.015.

2. Gach D, Nowak JU, Krzych 七. Epidemiology of out-of-hospital cardiac arrest in Bielsko-Biała district: a 12-month analysis. Kardiol Pol, 2016 May 25. doi: 10.5603/KP.a2016.0086.

3. Kudenchuk PJ, Stuart R, Husain S, Fahrenbruch C, Eisenberg M. Treatment and outcome of out-of-hospital cardiac arrest in outpatient health care facilities. Resuscitation, 2015; 97: 97-102. doi: 10.1016/ j.resuscitation.2015.08.025.

4. Talikowska M, Tohira H, Finn J. Cardiopulmonary resuscitation quality and patient survival outcome in cardiac arrest: A systematic review and meta-analysis. Resuscitation, 2015; 96: 66-77. doi: 10.1016/ j.resuscitation.2015.07.036.

5. Abella BS. High-quality cardiopulmonary resuscitation: current and future directions. Curr Opin Crit Care, 2016; 22(3): 218-224. doi: 10.1097/MCC.0000000000000296.

6. Cheng A, Hunt EA, Grant D et al. Variability in quality of chest compressions provided during simulated cardiac arrest across nine 
pediatric institutions. Resuscitation, 2015; 97: 13-19. doi: 10.1016/ j.resuscitation.2015.08.024.

7. Szarpak L, Filipiak KJ, Ładny JR, Smereka J. Should nurses use mechanical chest compression devices during cardiopulmonary resuscitation? Preliminary data. Am J Emerg Med, 2016 Jul 28. pii: S0735-6757(16)30478-8. doi: 10.1016/j.ajem.2016.07.057.

8. Kurowski A, Czyżewski L, Bogdański L Quality of chest compression with CardioPump CPR compared to single rescuer standard BLS. Am J Emerg Med, 2015; 33(1): 114-115. doi: 10.1016/j.ajem.2014.10.027.

9. Szarpak $Ł$, Truszewski Z, Smereka J, Czyżewski $Ł$. Does the use of a chest compression system in children improve the effectiveness of chest compressions? A randomized crossover simulation pilot study. Kardiol Pol, 2016 Jul 8. doi: 10.5603/KP.a2016.0107.

10. Kurowski A, Szarpak Ł, Bogdański Ł et al. Comparison of the effectiveness of cardiopulmonary resuscitation with standard manual chest compressions and the use of TrueCPR and PocketCPR feedback devices. Kardiol Pol, 2015; 73(10): 924-930. doi: 10.5603/KP.a2015.0084.

11. Wik L, Kramer-Johansen J, Myklebust $H$ et al. Quality of cardiopulmonary resuscitation during out-of-hospital cardiac arrest. JAMA, 2005; 293(3): 299-304.

12. Gässler H, Ventzke MM, Lampl L, Helm M. Transport with ongoing resuscitation: a comparison between manual and mechanical compression. Emerg Med J, 2013; 30(7): 589-592. doi: 10.1136/ emermed-2012-201142.

13. Soar J, Nolan JP, Böttiger BW et al. Section 3. Adult advanced life support. Resuscitation, 2015; 95: 100-147. doi: 10.1016/j.resuscitation.2015.07.016.

14. Xanthos T, Pantazopoulos I, Roumelioti H et al. A comparison of autopsy detected injuries in a porcine model of cardiac arrest treated with either manual or mechanical chest compressions. Eur J Emerg Med, 2011; 18(2): 108-110. doi: 10.1097/MEJ.0b013e32833e79cf.

15. Tranberg T, Lassen JF, Kaltoft AK et al. Quality of cardiopulmonary resuscitation in out-of-hospital cardiac arrest before and after introduction of a mechanical chest compression device, LUCAS-2; a prospective, observational study. Scand J Trauma Resusc Emerg Med, 2015; 23: 37. doi: 10.1186/s13049-015-0114-2.

16. Rubertsson $\mathrm{S}$. Update on mechanical cardiopulmonary resuscitation devices. Curr Opin Crit Care, 2016; 22(3): 225-229. doi: 10.1097/ MCC. 0000000000000302 .

17. Gässler H, Kümmerle S, Ventzke MM et al. Mechanical chest compression: an alternative in helicopter emergency medical services? Intern Emerg Med, 2015; 10(6): 715-720. doi: 10.1007/s11739-015-1238-0.

18. Truszewski Z, Szarpak L, Kurowski A et al. Mechanical chest compression with the LifeLine ARM device during simulated CPR. Am J Emerg Med, 2016; 34(5): 917. doi: 10.1016/j.ajem.2016.02.025.
19. McNeice AH, McAleavey NM, Menown IB. Advances in clinical cardiology. Adv Ther, 2014; 31(8): 837-860. doi: 10.1007/s12325014-0141-9.

20. Kalra A, Maharaj V, Johannsen RA, Hollenberg SM. Catheterization laboratory activation during mechanical cardiopulmonary resuscitation: when should we say "No?". Catheter Cardiovasc Interv, 2014; 83(1): 58-64. doi: 10.1002/ccd.25167.

21. Kleinman ME, Brennan EE, Goldberger ZD et al. Part 5: Adult Basic Life Support and Cardiopulmonary Resuscitation Quality: 2015 American Heart Association Guidelines Update for Cardiopulmonary Resuscitation and Emergency Cardiovascular Care. Circulation, 2015; 132(18 Suppl 2): S414-435. doi: 10.1161/CIR.00000000000000259.

22. Stiell IG, Brown SP, Christenson J et al. What is the role of chest compression depth during out-of-hospital cardiac arrest resuscitation? Crit Care Med, 2012; 40: 1192-1198.

23. Kurowski A, Czyżewski L, Bogdański L et al. Quality of chest compression with CardioPump CPR compared to single rescuer standard BLS. Am J Emerg Med, 2015; 33(1): 114-115. doi: 10.1016/ j.ajem.2014.10.027.

24. Peberdy MA, Silver A, Ornato JP. Effect of caregiver gender, age, and feedback prompts on chest compression rate and depth. Resuscitation, 2009; 80(10): 1169-74. doi: 10.1016/j.resuscitation.2009.07.003.

25. Sunde K, Wik L, Naess PA et al. Improved haemodynamics with increased compression-decompression rates during ACD-CPR in pigs. Resuscitation, 1998; 39(3): 197-205.

26. Fischer $\mathrm{H}$, Neuhold S, Zapletal B et al. A manually powered mechanical resuscitation device used by a single rescuer: a randomised controlled manikin study. Resuscitation, 2011; 82(7): 913-919. doi: 10.1016/ j.resuscitation.2011.02.026.

27. Edelson DP, Abella BS, Kramer-Johansen J et al. Effects of compression depth and pre-shock pauses predict defibrillation failure during cardiac arrest. Resuscitation, 2006; 71(2): 137-145.

28. Sell RE, Sarno R, Lawrence B et al. Minimizing pre- and post-defibrillation pauses increases the likelihood of return of spontaneous circulation (ROSC). Resuscitation, 2010; 81(7): 822-825. doi: 10.1016/ j.resuscitation.2010.03.013.

29. Putzer $G$, Braun P, Zimmermann A et al. LUCAS compared to manual cardiopulmonary resuscitation is more effective during helicopter rescue - a prospective, randomized, cross-over manikin study. Am J Emerg Med, 2013; 31(2): 384-389. doi: 10.1016/j.ajem.2012.07.018.

30. Putzer G, Fiala A, Braun P et al. Manual versus Mechanical Chest Compressions on Surfaces of Varying Softness with or without Backboards: A Randomized, Crossover Manikin Study. J Emerg Med, 2016; 50(4): 594-600. doi: 10.1016/j.jemermed.2015.10.002. 